\title{
Sustainable Layout Planning Requirements by Integration of Discrete Event Simulation Analysis (DES) with Life Cycle Assessment (LCA)
}

\author{
Victor Emmanuel de Oliveira Gomes ${ }^{1,2}$, Durval Joao De Barba Jr. ${ }^{1}$, \\ Jefferson de Oliveira Gomes ${ }^{1}$, Karl-Heinrich Grote ${ }^{2}$, and Christiane Beyer ${ }^{3}$ \\ ${ }^{1}$ Institute of Aeronautical Technology, Brazil \\ \{victor, debarbajr, gomes\}@ita.br \\ ${ }^{2}$ OvG University Magdeburg, Germany \\ \{victor.gomes, karl.grote\} eovgu. de \\ ${ }^{3}$ California State University Long Beach, USA \\ chris.beyer@csulb. edu
}

\begin{abstract}
Discrete Event Simulation (DES) provides computational models with different scenarios in which it is possible to check waste and capacityconstrained resources to generate comparative results. However, these analyses are guided by questions such as cost and production time without regard for wastes that impact on the environment. This paper examines how a tool of Life Cycle Assessment (LCA) can supplement DES in performing analyses of production systems taking into account environmental impacts, such as energy consumption and greenhouse emission. These ideas are demonstrated by a case study developed in an automotive company. The results ratify the importance of global projects with local solutions, even for layout planning.
\end{abstract}

Keywords: Discrete Event Simulation, Life Cycle Assessment, layout planning.

\section{Introduction}

A proper evaluation of manufacturing is a fundamental step to decision making in planning layouts. In these cases the support of mathematical tools, such as Discrete Event Simulation (DES), have been used for identifying waste on the shop floor and cost analyses for manufacturing optimization [1].

One of the advantages resulting from the application of DES in a corporate environment is its capability to include the impact of randomness in a system. All the dynamics and the non-deterministic nature of the parameters eliminate the use of static tools such as spreadsheets for solving many line design problems. Furthermore, all commercial simulation software provides detailed animation capabilities. The animation of the manufacturing process and flow can help engineers to visually detect problems or bottlenecks and also to test out alternate line designs. For these reasons DES may be applied to generate requirements and sustainable systems specifications 
for manufacturing. However, the analyses results realized by using DES are not sufficient for the joint assessment of impacts on the three dimensions of sustainability [2].

There are distinct tools and techniques to analyze and provide environmentally sustainable manufacturing systems. In most cases they consist of cost analyses integrated with pollutant emissions and energy efficiency analyses [3]. One of the biggest challenges for new production systems projects is obtaining data incorporated into the typical analyses of production (production capacity, material flow, transport, occupation rate of posts etc.)for the environmental impacts analysis. The Life Cycle Assessment (LCA) is a tool widely used in the academic environment and by corporations to calculate pollutant emissions rates. Through an LCA study it is possible to develop a systematic analysis of the environmental consequences associated with products during their life cycle, which improves the decision-making in areas such as innovation, regulations (industrial, environmental), strategies and policies.

This work discusses the combined use of DES with LCA to analyze production resource utilization in manufacturing systems. The combination of DES with LCA produces a powerful tool for analyzing the cause and effect of various scenarios where time, resources, place and randomness of input variables affect the outcome in sustainable manufacturing design. This joint application establishes the dynamic environment for sustainable production systems assessments and is an unexplored area addressed by few research publications. A case study was conducted, as a contribution towards this discussion, to analyze this joint use in decision-making for purchasing forklifts according to sustainable premises.

\section{Materials and Methods - Case Study}

The chosen problem for the proposed discussion was a sequencing process layout involving automotive door panels and it included situations concerning two important criteria contained in an industrial layout plan, which were transport performance and space. Transports cost can improve the efficiency of the product flow. It concerns the design of layout, the accommodation of people, and the machines and activities of a system or an enterprise within a physical spatial environment. Inefficient space utilization may cause cost increase and lead to competitive disadvantage in the market. As the result of less space demand in the planning phase, the company can either save rent cost or use the saved space for further development.

The system was a supermarket where automotive door panels, sequenced by the operators according to the customer's orders, were transported by forklift trucks until the dispatch sector. Each cell-sequencing had twelve containers overlapping with panels out of sequence from the assembly line, arranged in six sequenced pairs, and two containers with already sequenced panels, also overlapping. The production system goal was to provide a minimum amount of 380 containers daily in the dispatch area, with the minimum amount of operators and forklifts and reduced space 
utilization. From these premises, models with a pre-established configuration were simulated through DES software Plant Simulation-Tecnomatix ${ }^{\mathrm{TM}}$. A type of model is illustrated in Figure 1.

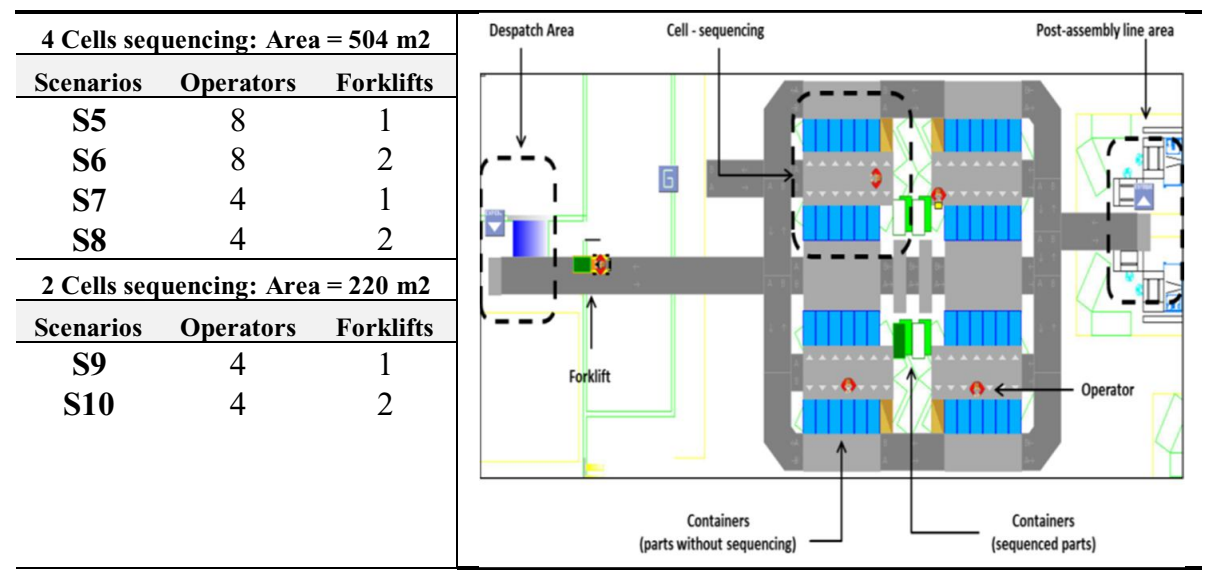

Fig. 1. - Computational model and proposed scenarios

For each proposed scenario the cost of forklift acquisition and personnel (labor/salary cost + incidental wage/salary cost - e.g. sickness, insurance fund, pension insurance fund etc.) was considered.

It is known that the usage phase of forklifts is of greatest environmental impact factors within its life cycle [4]. This high rate is directly related to the fuel consumption. In this way, the main factor to materially influence the choice of layout in forklift used must be the type of fuel used in addition to forklift performance. For this reason an LCA study was conducted in parallel with results supplied by the simulation analyses to verify the use of forklifts with different energy sources. Data from fuel utilization were acquired in different research sources.

This is a problem in which there are several objectives to be achieved simultaneously. Among the methods developed for evaluation in a multi-criteria decision environment the method PROMETHEE (Preference Ranking Organization Method for Enrichment Evaluation) was employed to support the decision-making process. It is appropriate in situations where decision-makers previously identified criteria and alternatives, by-passing structuring phase, and emphasizing the evaluation phase.

\section{Results and Discussion}

By the DES analysis, the scenario S9 was chosen due to better fulfillment of production requirements and the relation between cost and production with a lower shopfloor area (Figure 2). 


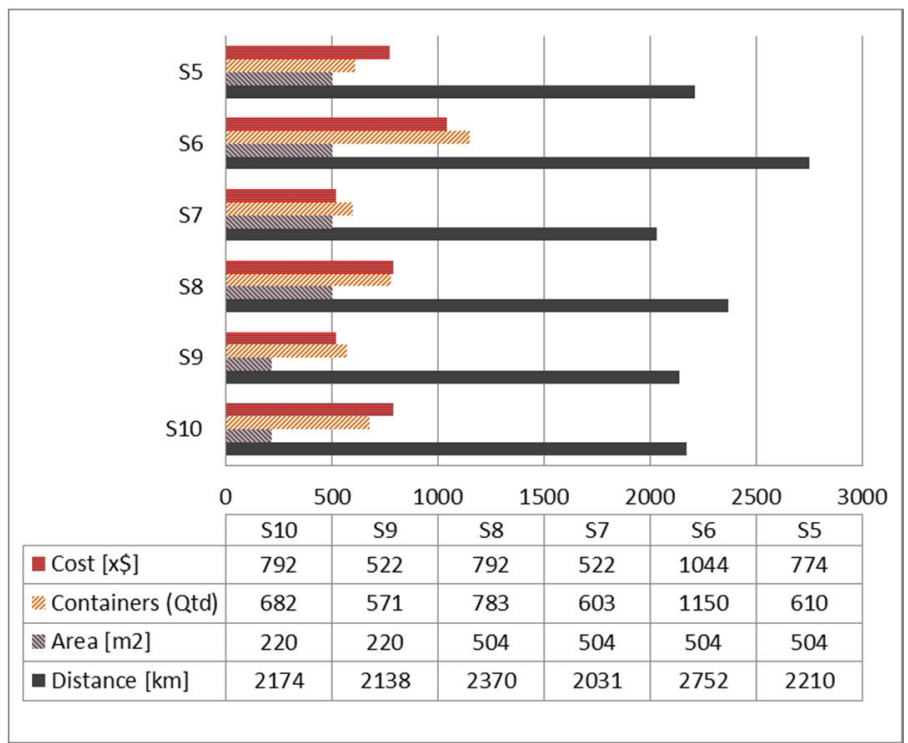

Fig. 2. - Proposed scenarios analyses

However, the scenario choices depends on the decision-making approach that can be done according to the area, the fuel consumption analysis, the forecast of future demands and other considerations which can form a decision-making matrix.

Based on [6-9] by the Lifecycle assessment, a comparison of five types of fuels used by forklifts was performed listing their environmental impacts due GWP (Global Warming Potential), displayed in metric tons $\mathrm{CO}_{2}$ equivalent per unit per year (ETC-Electricity Trickle Charge; EFC-Electricity Fast Charge; LPG- Liquefied Propane Gas; CNG-Compressed Natural Gas; Diesel and Gasoline) (Figure 3).

GWP (MtCO2e)

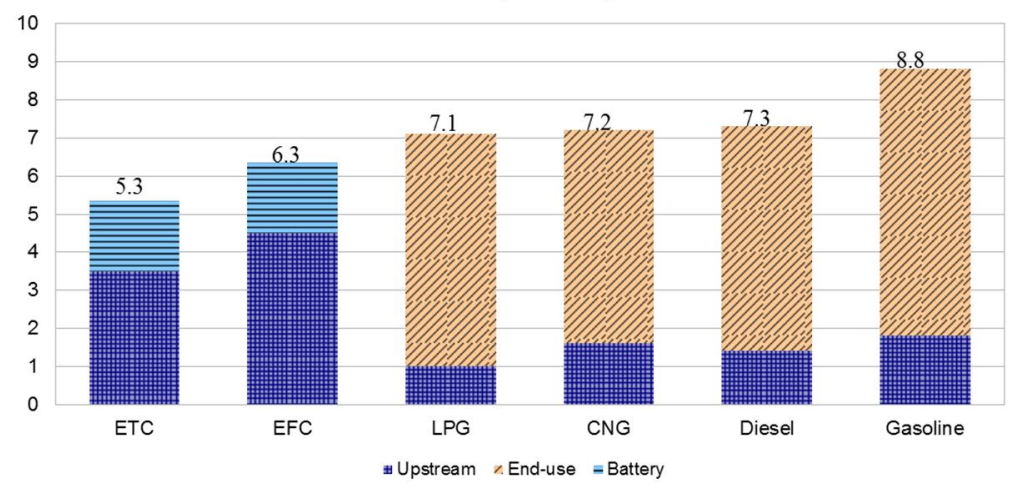

Fig. 3. - Comparison of fuels used by forklifts according GWP (Global Warming Potential): ETC-Electricity Trickle Charge; EFC-Electricity Fast Charge; LPG- Liquefied Propane Gas; CNG-Compressed Natural Gas; Diesel and Gasoline 
End-use emissions means the emission generated during the use of fuel. The meaning of upstream-emissions is related to emissions generated during the fuel production. The adopted term Battery means the emissions generated in the manufacturing, recycling and disposal phases of forklifts batteries.

A forklift needs 3 batteries daily. It was estimated a battery life of 50 months and also the influence of loading speed on the overall efficiency. Furthermore, a trickle charge (normal) presents an efficiency of $95 \%$ against $72 \%$ efficiency for a quick charge [5].

When the fuels are compared with the CNG, the Electricity Trickle Charge had the lowest impact $(-26.4 \%)$ followed by Electricity Fast Charge $(-12.5 \%)$. The Gasoline fuel had the most unsustainable impact $(+22.2 \%)$. The difference between LPG and Diesel fuels was very small, respectively $-1.4 \%$ and $+1.4 \%$.

Beyond this energy resource analysis, the Diesel fuel has to be considered as a particulate matter (PM) source harmful to human health impacting shop-floor area forklift use and leading to additional investments in ventilation systems.

Figure 4 presents the acquisition costs of forklifts. In comparison to CNG (normally used on shop-floors), Diesel propulsion presented the lowest value (-6\%), followed by electrical (-5.6\%). LPG and gasoline had much larger values, respectively $29.6 \%$ and $30.4 \%$. The electrical forklift analysis considered the cost for three batteries, including acquisition and maintenance for 50 months use.

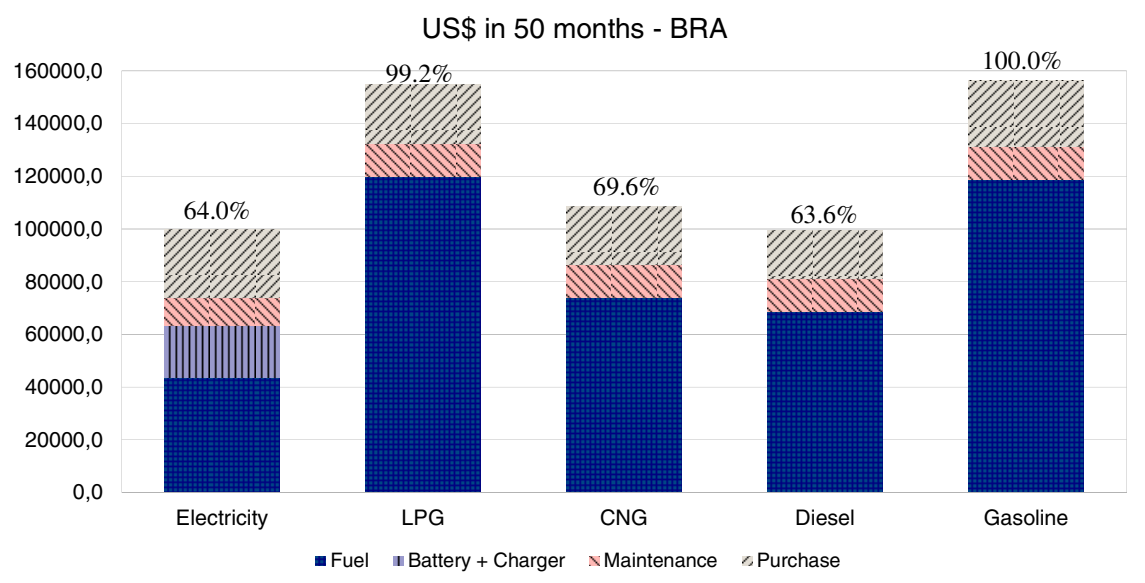

Fig. 4. - Acquisition costs of forklift

The presented results are based on the authors' premise that the environmental impact and cost analysis were the tasks to be considered in a decision making process for a sustainable shop-floor planning. However, both tasks were not significantly dependent, characterizing a need for a multi-criteria decision method (MDCA - Multi Decision Criteria Analysis).

In the application of PROMETHEE method, a MDCA tool, the authors considered weighted environmental impact and cost analysis $35 \%$ and $65 \%$ respectively. 
Using this method it presented a ranking of alternatives from the best to worst. Table 1 shows the sort of decision in accordance to the standards proposed by the decision maker.

Table 1. - PROMETHEE II Ranking

\begin{tabular}{l|cccccc}
\hline Alternatives & ETC & EFC & LPG & CNG & Diesel & Gasoline \\
\hline PROMETHEE II Ranking & 0.0724 & 0.0304 & -0.0060 & -0.0079 & -0.0115 & -0.0774 \\
Score & 100 & 91.92 & 85.47 & 85.13 & 84.52 & 74.06 \\
\hline
\end{tabular}

In accordance to the results, both electricity batteries were considered first choice. There were no significantly difference between LPG, CNG and Diesel. Gasoline fuel was the worst alternative. This analysis was considered for a land with high percentage of hydraulic generation, like Brazil and Norway.

However, considering a multinational company, which has global projects (there are differences in the amount of product demand and also areas occupied by production resources, different kinds of energy generation), the main idea is the consideration for applying local decisions.

In that case, considering the application of the MDCA method for a scenario in a land with high percentage of thermal generation, like USA, UK and Germany, the final results will be different than those for lands with a high percentage of hydraulic generation (Figure 5).

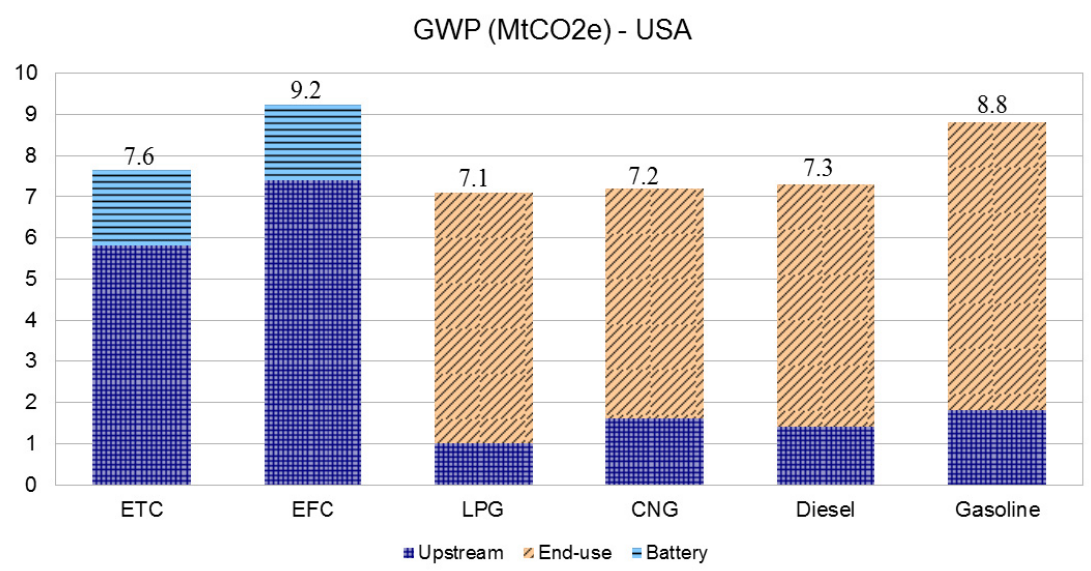

Fig. 5. - Comparison of fuels used by forklifts according GWP in lands with high percentage of thermal generation

For example, if the analysis had been conducted in the USA (percentage of hydraulic generation of $7.4 \%$ ), the results would be different than in Brazil (percentage of hydraulic generation of $83.2 \%$ ).

Similarly, the fuel costs are very different in those lands, being more expensive in Brazil. Electricity in Brazil is approximately three times greater than in USA [10]. 
For a MDCA analysis with the same alternatives, criteria and weights, a different ordering can be obtained. In this way the choice of the type of forklift in the USA would be preferably by LPG followed by CNG (Table 2).

Table 2. - PROMETHEE II Ranking for a new analysis considering lands with high percentage of thermal energy generation

\begin{tabular}{l|cccccc}
\hline Alternatives & LPG & CNG & Diesel & ETC & Gasoline & EFC \\
\hline PROMETHEE II Ranking & 0.0318 & 0.0301 & 0.0236 & 0.0113 & -0.0408 & -0.0559 \\
Score & 100 & 99.67 & 98.37 & 95.98 & 86.48 & 83.90 \\
\hline
\end{tabular}

It is observed that in this case there is a big difference between the forms of batteries, because the Fast Charge would be the worst choice (Figure 6).

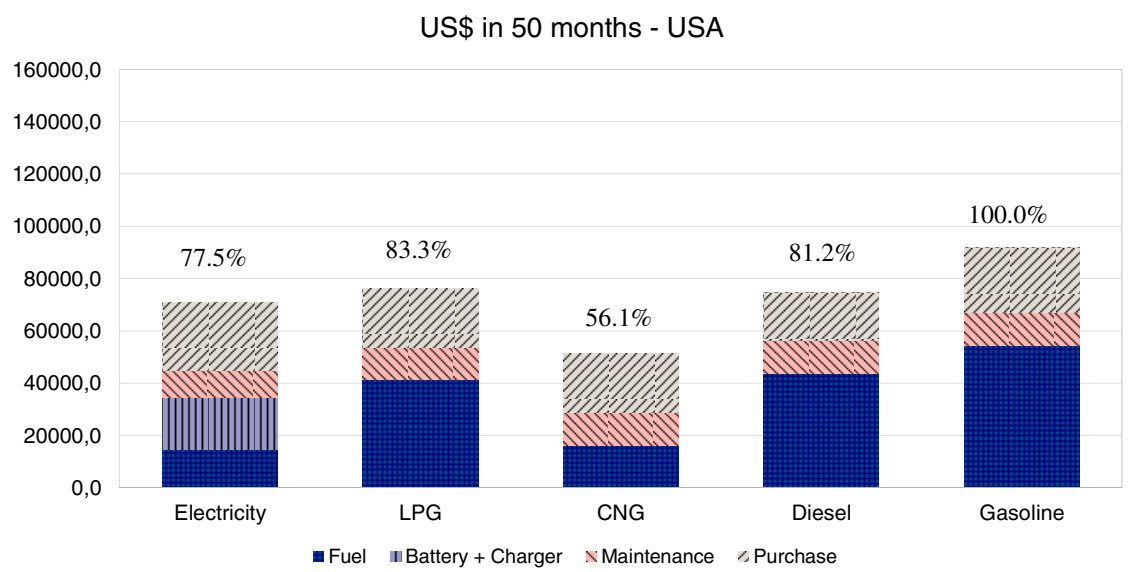

Fig. 6. - Comparison of fuels used by forklifts according dollars in 50 months in USA [10]

\section{Conclusions}

The combined use of DES and LCA presented a dynamic evaluation process for analyzing production resource in a sustainable manufacturing system environment.

Primarily, it was defined by simulation in a study case that assumed a production scenario combining small area, minimum amount of resources and efficient fuel consuming.

Supported by a Multi Criteria Decision Analysis Tool (PROMETHEE II), the use of forklifts was rationalized for distinct applications in shop-floors located in lands with high percentage of hydraulic and thermal energy generation.

The results ratify the importance of global projects with local solutions, even for layout planning. 


\section{References}

1. Standridge, C.R., Marvel, J.H.: Why Lean Needs Simulation. In: Winter Simulation Conference, pp. 1907-1913. Institute of Electrical and Electronics Engineers, Piscataway (2006)

2. Joschko, P., Page, B., Volker, W.: Combination of Job Oriented Simulation with Ecological Material Flow Analysis as Integrated Analysis Tool for Business Production Processes. In: Winter Simulation Conference (2009)

3. Helu, M., Rühl, J., Donfeld, D.: Evaluating Trade-Offs Between Sustainability, Performance, and Cost of Green Machining Technologies. In: Proceedings of the 18th CIRP International Conference on Life Cycle Engineering (2012)

4. Suzuki, N.: LCA Activities of Toyota Industries Corporation: Case Study. JLCA News Letter (2) (2006)

5. LPG AUSTRALIA. GHG life cycle assessment of LPG in the Australian stationary energy market. Report (49p) (August 2011)

6. Gaines, L.L., Elgowainy, A., Wang, M.Q.: Full Fuel-Cycle Comparison of Forklift Propulsion Systems (2008), http: / / greet.es.anl.gov/files/oh77n5k5

7. Antes, M., Brindle, R., McGevey, J., Pack, L., Zotter, B.: Propane's Greenhouse Gas Emissions: A Comparative Analysis. Propane Education \& Research Council (PERC). Report (39p) (2007)

8. Sullivan, J.L., Gaines, L.: A Review of Battery Life-Cycle Analysis: State of Knowledge and Critical Needs. Center for Transportation Research, Energy Systems Division, Argonne National Laboratory. Report (45p) (October 2010)

9. Wang, M.: Fuel Cycle Analysis of Conventional and Alternative Fuel Vehicles. Argonne National Laboratory. Encyclopedia of Energy, vol. 2.r, pp. 771-789. Elsevier Inc. (2004)

10. U.S. International Energy Agency (IEA): Key World Energy Statistics (2008) 